\title{
Erratum to: Ellipsoidal Inclusion with a Shell in an Anisotropic Medium Subjected to a Uniform Electric Field
}

\author{
I. V. Lavrov ${ }^{a, *}$ and V. B. Yakovlev ${ }^{a}$ \\ ${ }^{a}$ National Research University of Electronic Technology (MIET), Zelenograd, Moscow, 124498 Russia \\ *e-mail: iglavr@mail.ru \\ Received April 14, 2021
}

DOI: $10.1134 / \mathrm{S} 1063784221070100$

1. Page 1442, the first formula in (73) should read as follows:

$$
\begin{aligned}
\boldsymbol{\lambda}_{20}= & {\left[( \mathbf { I } + \mathbf { L } _ { 0 } ^ { ( 1 ) } ( \boldsymbol { \varepsilon } _ { 1 } \mathbf { I } - \boldsymbol { \varepsilon } _ { m } ) ) \left(\mathbf{I}+3^{-1} \varepsilon_{1}^{-1}\left(1-v^{\prime}\right)\right.\right.} \\
& \left.\left.\times\left(\boldsymbol{\varepsilon}_{2}-\boldsymbol{\varepsilon}_{1} \mathbf{I}\right)\right)+v^{\prime} \mathbf{L}_{0}^{(1)}\left(\boldsymbol{\varepsilon}_{2}-\boldsymbol{\varepsilon}_{1} \mathbf{I}\right)\right]^{-1},
\end{aligned}
$$

2. Page 1442 , the formula (75) should read as follows:

$$
\begin{gathered}
\mathbf{E}_{1}=\left(-\boldsymbol{\beta}^{(1)}+\varepsilon_{1}^{-1}\left[-3^{-1}\left(a^{(2)} / r\right)^{3} \mathbf{I}+\left(a^{(2)}\right)^{3}\right.\right. \\
\left.\left.\times r^{-5}(\mathbf{r} \otimes \mathbf{r})\right] \boldsymbol{\alpha}^{(1)}\right) \mathbf{E}_{0},
\end{gathered}
$$

The original article can be found online at https://doi.org/10.1134/S1063784218100158 